\title{
On the Equilibrium and Stability of ITER Relevant Plasmas
}

\author{
Ap Kuiroukidis ${ }^{1}$, G. N. Throumoulopoulos ${ }^{2 *}$, H. Tasso ${ }^{3}$ \\ ${ }^{1}$ Physics Department, University of Ioannina, GR 45110 Ioannina, Greece \\ ${ }^{2}$ Technological Education Institute of Serres, 62124 Serres, Greece \\ ${ }^{3}$ Max-Planck-Institut für Plasmaphysik, D-85748 Garching, Germany
}

Copyright (C)2015 by authors, all rights reserved. Authors agree that this article remains permanently open access under the terms of the Creative Commons Attribution License 4.0 International License

\begin{abstract}
We present recent results on steady states of translational symmetric and axisymmetric ITER relevant plasmas with incompressible sheared flow in connection with a generalized Grad-Shafranov (GGS) equation and on their stability. On the basis of a variety of ITER pertinent solutions of the GGS equation constructed it turns out that the flow affects noticeably the equilibrium quantities in qualitative agreement with the advanced confinement regimes phenomenology. Also, application of a sufficient condition for linear stability indicates that stabilization is mainly caused by the variation of the magnetic field in the direction perpendicular to the magnetic surfaces, related to the magnetic shear, depends on the plasma shaping and is sensitive to the up-down asymmetry. In certain cases the sheared flow has a weaker stabilizing effect enhanced by the equilibrium nonlinearity.
\end{abstract}

Keywords Magnetohydrodynamics, Equilibrium, Stability, Tokamak, Advanced Confinement

\section{Introduction}

It has been established in a number of fusion devices that sheared flows both zonal and mean (equilibrium) play a role in the transitions to improved confinement regimes as the L-H transition and the Internal Transport Barriers (ITBs). These flows can be driven externally in connection with electromagnetic power and neutral beam injection for plasma heating and current drive or can be created spontaneously (zonal flows). In the latter case the physical mechanism driving the flow is not completely understood; potential mechanisms are related among others to a residual stress, up-down asymmetry and the turbulence itself. Also, it remains physically rather unclear the role of the flow, no matter how is created, in the transitions to improved confinement regimes. The current experimental evidence and theoretical studies indicate that zonal flows in conjunction with externally driven ones suppress the turbulence thus contributing in the formation of transport barriers (cf the reviews $[1,2,3])$.

Equilibria with sheared flows which can be employed as starting points of stability and transport studies have been constructed on the basis of generalized GradShafranov equations (GGSs) [e.g. Eq. (1) below]. The stability of fluids and plasmas in the presence of equilibrium flows non parallel to the magnetic field remains a tough problem reflecting to the lack of necessary and sufficient conditions. Only for parallel flows few sufficient conditions for linear stability are available (e.g. [4]-[7]).

In an attempt to contribute to the understanding of the development of ipmroved confinement regimes in tokamaks, in this contribution we construct novel magnetohydrodynamic steady states and review recent ones [18]-[22] of translational symmetric and axisymmetric plasmas with incompressible sheared flow of arbitrary direction either with monotonically increasing safety factor profiles, pertinent to the L-H transition, or with reversed magnetic shear usually associated with the formation of ITBs. Specifically, linear and nonlinear solutions of the GGS equation (1) including non parallel flows of plasmas surrounded by a diverted ITER pertinent boundary have been constructed analytically, quasianalytically and numerically. The equilibrium characteristics in connection with the improved confinement regimes phenomenology are also examined. Furthermore, for flows parallel to the magnetic field we examine the linear stability of the equilibria constructed by means of the sufficient condition of [6].

The generalized Grad-Shafranov equation is briefly reviewed in Sec. 2 where also solutions are presented, including a novel ITER pertinent one, together with certain equilibrium characteristics. The stability of equilibria with parallel flows are examined on the basis of these solutions in Sec. 3 and Sec. 4 summarizes the main conclusions. 


\section{Solutions and equilibrium char- acteristics}

The equilibrium of an axisymmetric plasma with incompressible flow satisfies the generalized GradShafranov equation $[8,9,10]$

$$
\begin{array}{r}
\Delta^{*} u+\frac{1}{2} \frac{d}{d u}\left(\frac{X^{2}}{1-M_{p}^{2}}\right)+\mu_{0} R^{2} \frac{d P_{s}(u)}{d u} \\
+\mu_{0} \frac{R^{4}}{2} \frac{d}{d u}\left[\varrho\left(\frac{d \Phi}{d u}\right)^{2}\right]=0
\end{array}
$$

and the Bernoulli equation for the pressure

$$
P=P_{s}-\varrho\left[\frac{v^{2}}{2}-R^{2}\left(\frac{d \Phi}{d u}\right)^{2}\right]
$$

Here, $u(R, z)$ is the poloidal magnetic flux function [with $(z, R, \phi)$ cylindrical coordinates with $z$ corresponding to the axis of symmetry], $M_{p}(u)$ the Mach function of the poloidal velocity with respect to the poloidal-magneticfield Alfvén velocity, $P_{s}(\psi)$ the pressure in the absence of flow, $\Phi(u)$ the electrostatic potential, $\varrho(u)$ the plasma density, $X(u)$ relates to the toroidal magnetic field and $\Delta^{\star}:=R^{2} \nabla \cdot\left(\nabla / R^{2}\right)$. Derivation of $(1)$ and $(2)$ is provided in $[8,9,10]$. Once the free functions $M_{p}(u), P_{s}(u)$, $\Phi(u), \varrho(u)$ and $M_{p}(u)$ are assigned, Eq. (1) can be solved under appropriate boundary conditions. The respective GGS equation for a translational symmetric plasma is given by Eq. (8) of [9].

We have solved Eq. (1) in three alternative ways: i) by constructing analytic solutions including extensions of the Solovév solution, the generic linear solution of [11] and the nonlinear solution of [12], ii) by using appropriate ansatz for the unknown poloidal magnetic flux function which reduces the original equation to a set of ODEs and algebraic constraints; then this set of equations is solved numerically, and iii) fully numerically by prescribing analytically either a D-shaped or a diverted boundary. Here we present a new numerical solution for parallel flow $\left(\Phi^{\prime}(u) \equiv 0\right)$ constructed as follows. First, we make the normalizations $\rho=R / R_{0}$, $\zeta=z / R_{0}$, where $R_{0}$ is the major radius of the configuration; $\tilde{u}=u / u_{0}, \tilde{P}_{s}=P_{s} / P_{0}, \tilde{X}=X / X_{0}$, where $X_{0}:=u_{0} / R_{0}, u_{0}^{2}:=P_{0} \mu_{0} R_{0}^{4}$ and $P_{0}$ is a reference pressure. Eq. (1) is then written in the dimensionless form

$$
\begin{aligned}
{\left[\partial_{\rho \rho}-(1 / \rho) \partial_{\rho}+\partial_{\zeta \zeta}\right] \tilde{u} } & +\frac{1}{2} \frac{d}{d \tilde{u}}\left[\frac{\tilde{X}^{2}}{1-M_{p}^{2}}\right]+ \\
& +\rho^{2} \frac{d \tilde{P}_{s}}{d \tilde{u}}=0
\end{aligned}
$$

Choosing the free-function terms as

$$
\begin{gathered}
\frac{\tilde{X}^{2}}{\left(1-M_{p}^{2}\right)}=\left[\tilde{X}_{0}^{2}+2 \tilde{X}_{1} \tilde{u}+\tilde{X}_{2} \tilde{u}^{2}+\frac{2}{3} \tilde{X}_{3} \tilde{u}^{3}\right], \\
\tilde{P}_{s}(\tilde{u})=\tilde{P}_{0} \exp \left[V^{2}\left(\frac{1}{\left(\tilde{u}_{a}-\tilde{u}_{b}\right)^{2}}-\frac{1}{\left(\tilde{u}-\tilde{u}_{b}\right)^{2}}\right)\right],
\end{gathered}
$$

Eq. (3) assumes the form

$$
\begin{aligned}
{\left[\rho \frac{\partial}{\partial \rho}\left(\frac{1}{\rho} \frac{\partial}{\partial \rho}\right)+\frac{\partial^{2}}{\partial \zeta^{2}}\right] \tilde{u} } & +\left(\tilde{X}_{1}+\tilde{X}_{2} \tilde{u}+\tilde{X}_{3} \tilde{u}^{2}\right)+ \\
+ & \rho^{2}\left(\frac{d \tilde{P}_{s}}{d \tilde{u}}\right)=0
\end{aligned}
$$

Here, $\tilde{u}_{a}\left(\tilde{u}_{b}\right)$ is the normalized poloidal flux function on the boundary (magnetic axis); $\tilde{X}_{0}, \tilde{X}_{1}, \tilde{X}_{2}, \tilde{X}_{3}, \tilde{P}_{0}$, and $V$ are dimensionless constants. We prescribe analytically a fixed boundary with a lower X-point by the parametric equations (17)-(20) of [21] and solve numerically Eq. (6 ) by adopting the nine-point-formula integration scheme described in detail in Section 4 of our previous paper [21]. Also, we assign the following parametric values: $R_{0}=6.2 \mathrm{~m}, P_{0}=1.6 \times 10^{5} \mathrm{~Pa}=1.58 \mathrm{Atm}$ implying that $X_{0}=2.78 \mathrm{~Wb} / \mathrm{m}$ and $u_{0}=17.23 \mathrm{~Wb}$. An ITER-like configuration in connection with this solution is given in Fig. 1. The safety factor of this equilibrium

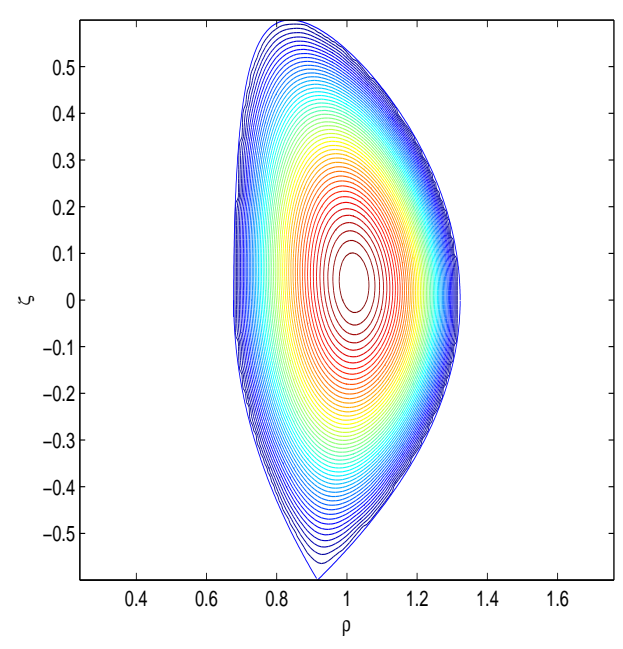

Figure 1. ITER pertinent numerical equilibrium solution in connection with the choice (4) and (5) for major radius, $R_{0}=6.2$ $\mathrm{m}$, minor radius, $a=2 \mathrm{~m}$, elongation, $\kappa=1.64$ and triangularity, $\delta=0.5$. The bounding flux surface corresponds to $\tilde{u}_{b}=1 \mathrm{~Wb}$, with $\tilde{u}_{a}=1.23$ for the magnetic axis.

increases monotonically from the magnetic axis to the boundary. A stability consideration in connection with the L-H transition is made in Sec. 3. The solution holds for arbitrary Mach functions, $M_{p}^{2}(u)$, and densities, $\varrho$. To completely, construct the equilibrium we have made several choices of these functions, e.g.

$$
\begin{aligned}
\varrho(u) & =\varrho_{a}\left(1-\frac{u^{2}}{u_{b}^{2}}\right)^{\lambda} \\
M_{p}^{2} & =M_{a}^{2}\left[1-\left(\frac{u}{u_{b}}\right)\right]^{n}, \\
M_{p}^{2} & =M_{0}^{2}\left(\tilde{u}_{a}-\tilde{u}\right)^{m}\left(\tilde{u}-\tilde{u}_{b}\right)^{n}
\end{aligned}
$$

Here, the free parameters $\varrho_{a}$ and $M_{a}^{2}$ correspond to the maximum values of $\varrho$ and $M_{p}^{2}, M_{0}^{2}$ relates to the maximum value of $M_{p}^{2} ; m$ and $n$ are connected to the flow shear. In particular, (8) is peaked on- while (9) is peaked off-axis in connection with respective auxiliary heating of tokamaks. For the equilibrium of Fig. 1 we have chosen $M_{0}^{2}=0.01$, and $m=n=2$. 
More general the equilibria constructed here and in our past studies [17]-[22] have either safety factor profiles monotonically increasing from the magnetic axis to the plasma edge pertinent to the L-H transition or reversed magnetic shear related to the formation of ITBs. The equilibrium characteristics have been examined by means of the pressure, safety factor $(q)$, current density, toroidal velocity, poloidal Mach function and electric field (E) associated with the non parallel flow. It turns out that:

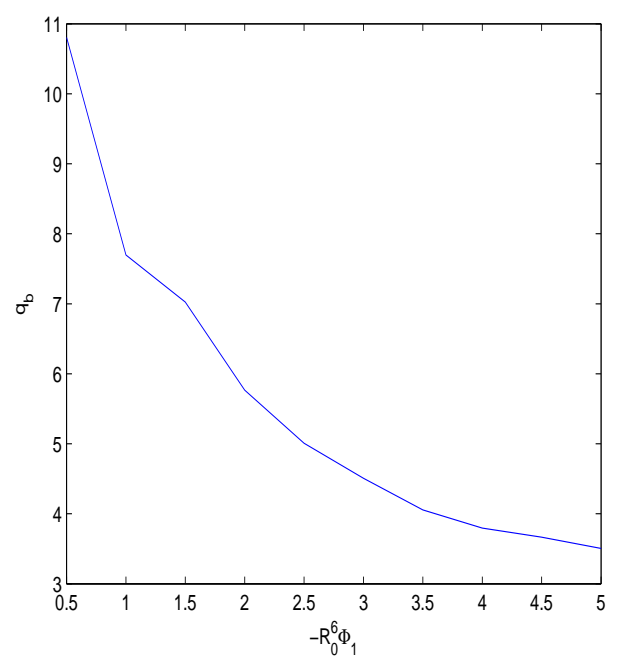

Figure 2. Dependence of the edge (boundary) safety factor $q_{b}$ on an electrostatic potential parameter $\Phi_{1}$, with $q_{a}=1$, for an anlytic solution of (1) constructed in [18] [Eqs. (22) and (23) therein]. The curve indicates that the $q$-profile becomes flatter as $\left|\Phi_{1}\right|$ increases

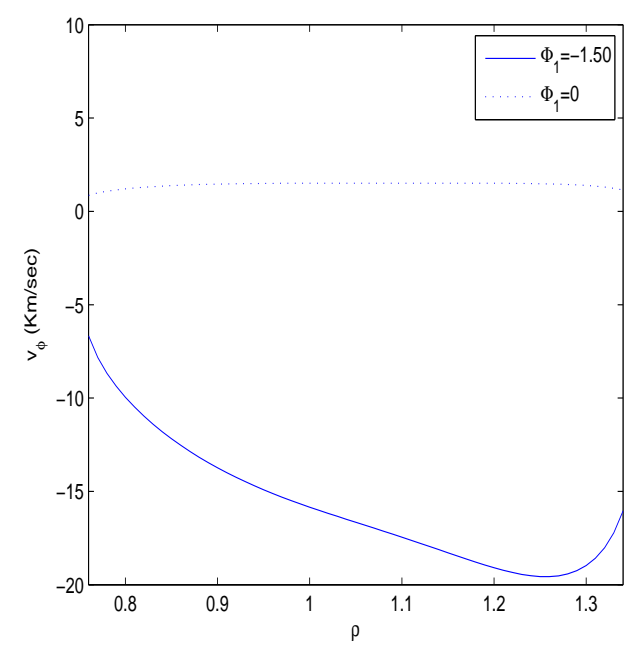

Figure 3. Toroidal velocity for an analytic solution of (1) constructed in [18] [Eqs. (22) and (23) therein] with different values of an electrostatic-potential parameter $\Phi_{1}$ and $q_{a}=1$. The graphs indicate that the larger $\left|\Phi_{1}\right|$ is the higher the amplitude and shear of $v_{\phi}$

- E makes the $q$-profile flatter (Fig. 2) and increases the magnitude and shear of the toroidal velocity (Fig. 3) in qualitative agreement with experimental evidence on the formation of ITBs, thus indicating a stabilizing effect of the electric field.
- The maximum of $\mathbf{E}$ takes larger values as the flow amplitude increases but its position is insensitive to the flow.

- The results indicate stabilization by synergetic effects of $\mathbf{E}$ and magnetic shear. A synergism of reversed magnetic shear and sheared poloidal and toroidal rotation, consisting in that on the one hand the reversed magnetic shear plays a role in triggering the ITBs development while on the other hand the sheared rotation has an impact on the subsequent growth and allows the formation of strong ITBs, was observed in JET [13] and DIII-D [14].

\section{Stability Consideration}

We now consider the linear stability of the equilibria constructed for field aligned flows by applying the sufficient condition of [6]. This condition states that a stationary equilibrium with field aligned incompressible flow and constant mass density is stable to small three dimensional perturbations if $M_{p}^{2}<1$ and $A \geq 0$ where

$$
\begin{aligned}
A & =A_{1}+A_{2}+A_{3}+A_{4} \\
A_{1} & =-(\mathbf{J} \times \nabla u)^{2} \\
A_{2} & =(\mathbf{J} \times \nabla u) \cdot(\nabla u \cdot \nabla) \mathbf{B} \\
A_{3} & =-\frac{1}{4}\left(1-M_{p}^{2}\right)^{-1} \frac{d M_{p}^{2}}{d u}|\nabla u|^{2} \nabla u \cdot \nabla B^{2} \\
A_{4} & =-\frac{1}{2}\left(1-M_{p}^{2}\right)^{-3 / 2} \frac{d M_{p}^{2}}{d u}|\nabla u|^{4} g \\
g & =\frac{1}{\sqrt{1-M_{p}^{2}}}\left(\frac{d P_{s}}{d u}-\frac{d M_{p}^{2}}{d u} \frac{B^{2}}{2}\right)
\end{aligned}
$$

The quantities in(10)-(15) are dimensionless with the tilde dropped for notation simplicity. The quantity $A_{1}$ being always negative consists a destabilizing contribution potentially related to current driven modes. The other terms can be either stabilizing or destabilizing. Specifically, the term $A_{2}$ relates to the current density and the variation of the magnetic field perpendicular to the magnetic surfaces. The term $A_{3}$ involves the shear and magnitude of the flow in conjunction with the variation of the magnitude of the magnetic field perpendicular to the magnetic surfaces. $A_{4}$ is mostly a flow term depending on the magnitude and the shear of the flow. The flows satisfying (1) are inherently sub-Alfvénic $\left(M_{p}^{2}<1\right)$ because of an integral transformation involved [9]. To apply the condition we set in Eq. (1) $\Phi^{\prime} \equiv 0$ and $\varrho=\varrho_{a}=1$. Also, $M_{p}$ is now the Alfvén Mach function of the parallel velocity because in this case it can be shown that the poloidal Mach function, the respective toroidal Mach function and the (total) parallel Mach function are equal one another.

Application of the condition $A \geq 0$ for a variety of the equilibria constructed led to the following conclusions.

- We have identified a potentially stable equilibrium, in the sense that the stability condition is satisfied in an appreciable part of the plasma, with high flow shear corresponding to the H-state (Fig. 7), while another equilibrium with low flow shear corresponding to the L-state does not satisfy the condition (see also the foorth item below). 


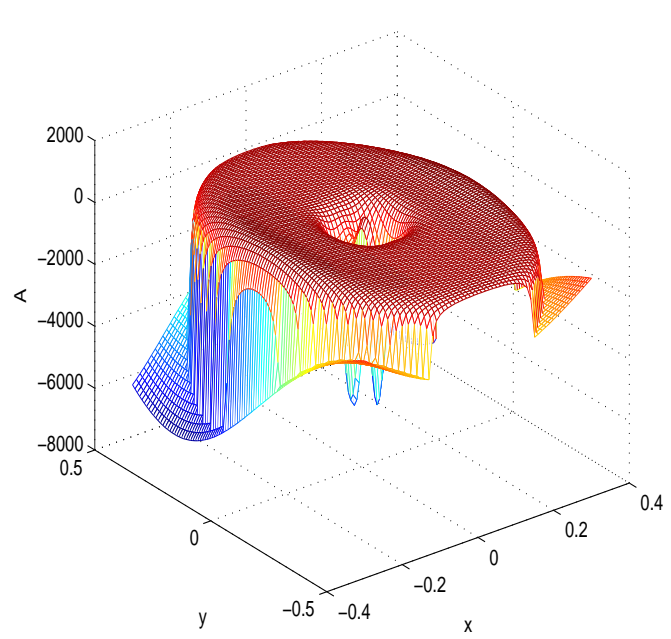

Figure 4. Two-dimensional plot of the stability quantity $A$ for a translational symmetric equilibrium solution [19] with parallel flow. For most part of the plasma domain $A$ is non-negative and assumes negative values only in the core region.

- Stabilization is mainly caused by the term $A_{2}$ related to the magnetic shear.

- Depending on the Mach-function profiles and the values of the free parameters the flow and flow shear may have rather weak stabilizing effects. It is clarified, however, that this is far from a "universal" result; there are cases of flow and flow shear destabilization.

- In certain cases stability is improved by the equilibrium nonlinearity in agreement with past equilibrium studies in plane geometry $[15,16]$. A pertinent example supporting this result in connection with the equilibrium of Fig. 1 is presented in Figs. 5-7. Fig. 5 refers to a static equilibrium $\left(M_{0}=0\right)$ for the value of the nonlinear parameter $V=1.5$ for which the stability condition $A \geq 0$ is nearly nowhere satisfied. We conjecture that this corresponds to the L state. In the stability graph of Fig. 6 associated with a stronger equilibrium nonlinearity $(V=2.2)$ and off-axis flow [Eq. (9)] the condition $A \geq 0$ is satisfied in a major part of the plasma indicated by the red-colored region. This may correspond to the $\mathrm{H}$ state. Respective curves of the quantity $A$ on the plane $z=0$ are given in Fig. 7 .

- The stability is affected by the plasma shaping and is sensitive to even small up-down asymmetry.

According to the above results and the believe that the sheared flow is developed during the L-H transition we conjecture that a static equilibrium could correspond to the L state and a stationary equilibrium with flow shear and stronger nonlinearity to the $\mathrm{H}$ state. In a quasistatic evolution approximation the plasma then potentially evolves through successive states with increased equilibrium nonlinearity and sheared flow. A similar process may be relevant to the formation of Internal Transport Barriers according to the results of [22].

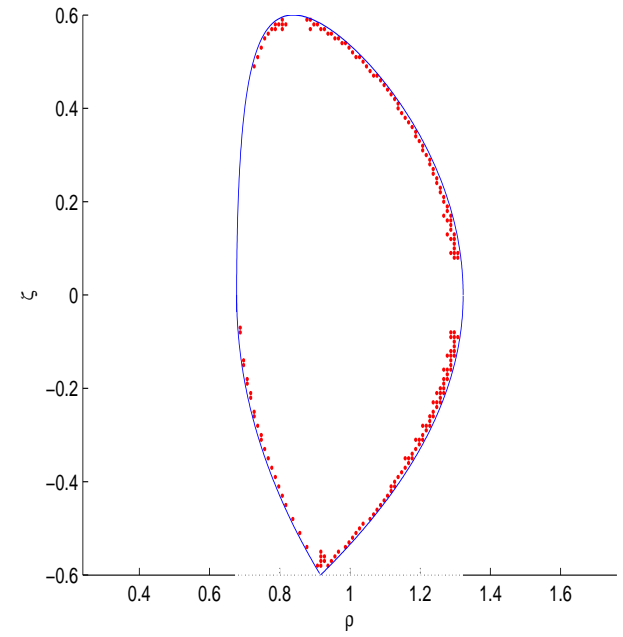

Figure 5. Stability graph for the equilibrium of Fig. 1 corresponding to the L-sate for $M_{0}=0$ and $V=1.5$ showing the sign of the stability quantity $A$. Only in the minor red colored spots it holds $A \geq 0$ and therefore this condition is nearly nowhere satisfied.

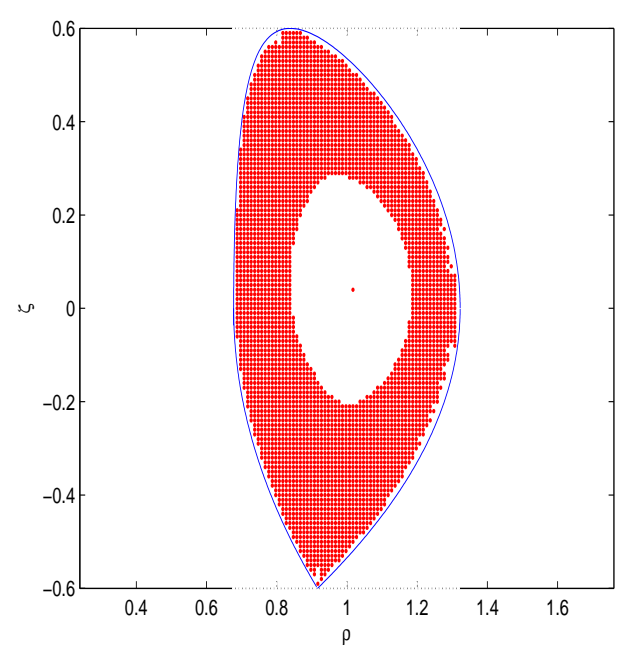

Figure 6. Stability graph for the equilibrium of Fig. 1 corresponding to the H-state with strong equilibrium nonlinearity $(V=2.2)$ and sheared flow $\left(M_{0}^{2}=0.01\right.$ and $\left.m=n=2\right)$. The stability condition $A \geq 0$ is satisfied in the red-colored region.

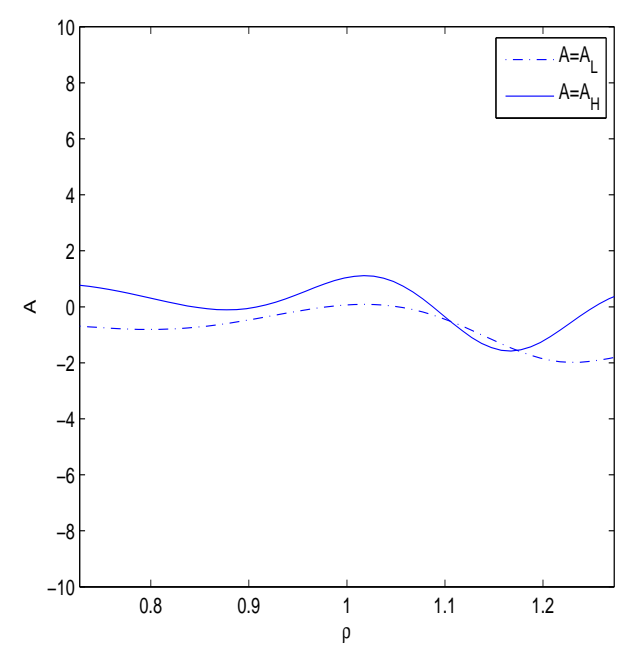

Figure 7. Plots of the stability quantity $A$ on the plane $z=0$ for the configuration of Fig. 1. The dashed-doted curve corresponds to the L-state while the continuous one to the H-state. 


\section{Conclusions}

In a series of recent studies [17]-[20] and here we have constructed linear and nonlinear equilibria either with monotonically increasing safety factor profiles or with reversed magnetic shear. For non parallel flows it turns out that the electric field makes the safety factor flatter and increases the magnitude and shear of the toroidal velocity in qualitative agreement with experimental evidence on the formation of ITBs in tokamaks, thus indicating a potential stabilizing effect of the electric field. For parallel flows application of a sufficient condition for linear stability implies that the variation of the magnetic field perpendicular to the magnetic surfaces has a stabilizing effect with a possible weaker contribution from the sheared flow. The results indicate that depending on the Mach function profile and the values of the free parameters the sheared flow in conjunction with the equilibrium nonlinearity can play a stabilizing role and that the stability is affected by the up-down asymmetry.

\section{Acknowledgements}

GNT wishes to thank George Poulipoulis and Alexander Nindos for useful discussions. This work has been carried out within the framework of the EUROfusion Consortium and has received funding from (a) the National Programme for the Controlled Thermonuclear Fusion, Hellenic Republic, (b) Euratom research and training programme 2014-2018 under grant agreement No 633053. The views and opinions expressed herein do not necessarily reflect those of the European Commission.

\section{REFERENCES}

[1] K. Itohy and S-I Itoh, Plasma Phys. Control. Fusion 38, 1 (1996).

[2] R. C. Wolf, Plasma Phys. Control. Fusion 45, R1-R91 (2003).

[3] C. D. Challis, Plasma Phys. Control. Fusion 46, B23 (2004).

[4] S. Friedlander, M.M. Vishik, Chaos 5, 416 (1995).

[5] V. A. Vladimirov and K. I. Ilin, Phys. Plasmas 5, 4199 (1998).

[6] G. N. Throumoulopoulos and H. Tasso, Phys. Plasmas 14, 122104 (2007).
[7] P. J. Morrison, E. Tassi, N. Tronko, Phys. Plasmas 20 042109 (2013).

[8] H. Tasso and G. N. Throumoulopoulos, Phys. Plasmas 5, 2378 (1998).

[9] C. Simintzis, G. N. Throumoulopoulos, G. Pantis, and H. Tasso, Phys. Plasmas 8, 2641 (2001).

[10] G. N. Throumoulopoulos, H. Tasso, G. Poulipoulis, J. Plasma Physics 74, 327 (2008).

[11] C. V. Atanasiu, S. Gunter, K. Lackner, and I. G. Miron, Phys. Plasmas 11, 3510 (2004).

[12] G. Cicogna, F. Pegoraro and F. Ceccherini, Phys. Plasmas 17, 102506 (2010).

[13] P. C. de Vries, E. Joffrin, M. Brix et al., Nucl. Fusion 49, 075007 (2009).

[14] M. W. Shafer, G. R. McKee, M. E. Austin et al., Phys. Rev. Lett. 103, 075004 (2009).

[15] G. N. Throumoulopoulos, H. Tasso and G. Poulipoulis, J. Phys. A: Math. Theor. 42, 335501 (2009).

[16] G. N. Throumoulopoulos and H. Tasso Phys. Plasmas 17, 032508 (2010).

[17] G. N. Throumoulopoulos, H. Tasso, Phys. Plasmas 19 , 014504 (2012).

[18] Ap Kuiroukidis G. N. Throumoulopoulos, Phys. Plasmas 19, 19, 022508 (2012).

[19] Ap Kuiroukidis and G. N. Throumoulopoulos, J. Plasma Physics 79, 257 (2013).

[20] Phys. Plasmas 21, 032509 (2014); Plasma Phys. Control. Fusion 56, 075003 (2014).

[21] Ap Kuiroukidis and G. N. Throumoulopoulos, J. Plasma Physics 80, 27 (2014).

[22] Ap Kuiroukidis and G. N. Throumoulopoulos, J. Plasma Physics 81, 905810404 (2015). 